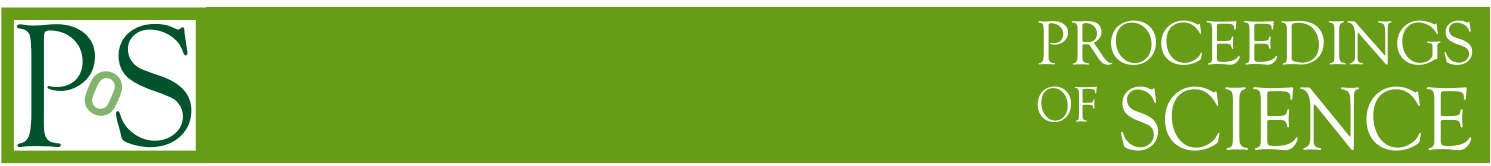

\title{
Measurements of vector boson fusion with the ATLAS detector
}

\author{
P. Calfayan*, on behalf of the ATLAS Collaboration \\ Indiana University \\ E-mail: philippe.calfayan@cern.ch
}

The ATLAS experiment at the LHC performed measurements of the production of $\mathrm{W}$ and $\mathrm{Z}$ bosons in association with two jets at high dijet invariant mass. Results are based on proton-proton collisions at a center-of-mass energy of $\sqrt{s}=7,8$, and $13 \mathrm{TeV}$, with an integrated luminosity of 4.7, 20, and $3.2 \mathrm{fb}^{-1}$, respectively. Integrated and differential cross sections are measured in multiple regions of the phase space, with different sensitivities to the electroweak production via vector boson fusion. Unfolded differential cross sections are provided and compared to theoretical predictions. The results are also further used to constrain anomalous gauge couplings.

XXVI International Workshop on Deep-Inelastic Scattering and Related Subjects (DIS2018)

16-20 April 2018

Kobe, Japan

${ }^{*}$ Speaker. 


\section{Introduction}

At the LHC, the production of a $\mathrm{W}$ or $\mathrm{Z}$ boson in association with two jets ( $\mathrm{Wjj}$ or $\mathrm{Zjj}$ ) can be achieved by electroweak (EW) or strong (QCD) processes. The EW production consists in the exchange of an electroweak boson in the t-channel mode, including the fusion of vector bosons (VBF). The $\mathrm{Wjj}$ and Zjj final states are however dominantly obtained via QCD processes.

The EW and QCD productions of $\mathrm{Wjj}$ and $\mathrm{Zjj}$ events have been measured by the ATLAS experiment [1] in signal enriched fiducial regions. Differential cross sections have also been computed and unfolded in the case of various observables which discriminate between EW and QCD processes.

The EW VBF production process is of particular interest as it is sensitive to anomalous triplegauge-boson couplings (WWZ and WW $\gamma$ ). In addition, VBF signatures are important to measure couplings to the Higgs boson, enable searches for new particles beyond the Standard Model (SM), and can be used to tune Monte Carlo (MC) simulation.

\section{Measurement of the $\mathrm{Wjj}$ production cross section}

The $\mathrm{Wjj}$ production cross sections in the electron and muon decay channels have been measured and combined, using 4.7 and $20 \mathrm{fb}^{-1}$ of data at 7 and $8 \mathrm{TeV}$ [2], respectively.

The signal region (SR) consists in events including a W boson candidate, two leading jets well separated in rapidity with transverse momenta $\left(p_{\mathrm{T}}\right)$ exceeding 80 and $60 \mathrm{GeV}$, and an associated dijet invariant mass larger than $500 \mathrm{GeV}$. In addition, the signal lepton is required to be central with respect to the two leading jets, and events with an additional central jet are vetoed.

The main background of the analysis is the QCD production of $\mathrm{Wjj}$ events, which constitute nearly $60 \%$ of the SR, while the multijet and top processes each amounts to approximately $10 \%$ of the SR. In order to minimize the uncertainty on the simulation of the QCD Wjj production from POWHEG+PYTHIA8, the expectation is constrained with data events from a control region (CR) based on SR cuts, yet with the signal lepton required to be forward. The multijet background is estimated from data using a template enriched in multijet events.

The final normalizations in the SR of the CR-corrected QCD-Wjj events and the EW-Wjj signal simulated by POWHEG+PYTHIA 8 are obtained via a global fit to data of the dijet invariant mass. The resulting distribution is shown in Figure 1. The fiducial cross sections derived in the $\mathrm{SR}$ for the EW Wjj production are $144 \pm 35 \mathrm{fb}$ and $159 \pm 25 \mathrm{fb}$ at 7 and $8 \mathrm{TeV}$, respectively. The largest experimental uncertainty at $8 \mathrm{TeV}$ is due to the calibration of the jets, and is of nearly $8 \%$.

Fiducial cross sections measurements of the EW and EW plus QCD productions have been performed in multiple regions, as illustrated in Figure 2, together with the associated theoretical predictions.

\section{Measurement of the $\mathrm{Zjj}$ production cross section}

Both the electron and muon decay channels of the $\mathrm{Zjj}$ production have been analyzed and combined using 20 and $3.2 \mathrm{fb}^{-1}$ of data at 8 [3] and $13 \mathrm{TeV}$ [4], respectively. 
Similarly to the Wjj analysis, the nominal SR (EW-enriched) is defined by events including a $\mathrm{Z}$ boson candidate, a dijet invariant mass exceeding $250 \mathrm{GeV}$, and no additional jets in the rapidity gap between the two leading jets. However, looser lower thresholds on the two leading jets are required, namely 45 and $55 \mathrm{GeV}$, in order to keep sufficient statistics. In addition, the modulus of the vectorial sum of the two leading leptons and jets over the sum of the modulus of the separate objects is constrained to values below 0.15 , which suppresses pileup or multiple parton interactions, as well as poorly measured jets.

The dominant background of the analysis is the QCD production of $\mathrm{Zjj}$ events, which is simulated by SHERPA V1.4.3 for the $8 \mathrm{TeV}$ analysis, and either MADGRAPH_aMC@NLO+PYTHIA8, SHERPA V2.2.1, or ALPGEN+PYTHIA 6 for the $13 \mathrm{TeV}$ analysis. The estimation of the QCD$\mathrm{Zjj}$ production is constrained using data events from a QCD-enriched CR. The latter differs from the SR in that at least one jet is required to be within the rapidity gap between the two leading jets.

The EW-Zjj signal is predicted by SHERPA V1.4.3 for the $8 \mathrm{TeV}$ analysis, and either POWHEG+PYTHIA8 or SHERPA V2.2.1 for the $13 \mathrm{TeV}$ analysis. The CR-corrected QCD-Zjj events and the simulated signal are scaled to match data in the SR via a global fit of the dijet invariant mass, which is illustrated in Figure 3. In the specific case of the measurement at $13 \mathrm{TeV}$, the final normalizations of the EW-Zjj signal and the QCD-Zjj background are derived from the average of the six fits obtained from all combinations of the different QCD-Zjj and EW-Zjj simulations. The fiducial cross sections of the $\mathrm{Zjj}$ production determined in the SR are $54.7_{-10.9}^{+11.5} \mathrm{fb}$ and $119 \pm 26 \mathrm{fb}$ at 8 and $13 \mathrm{TeV}$, respectively. The largest uncertainty, which is due to the modelling of the shape of the dijet invariant mass, reaches $11 \%$.

Fiducial cross sections measurements of the EW and EW plus QCD productions have been computed in several regions, as provided in Figure 4, together with the associated theoretical predictions.

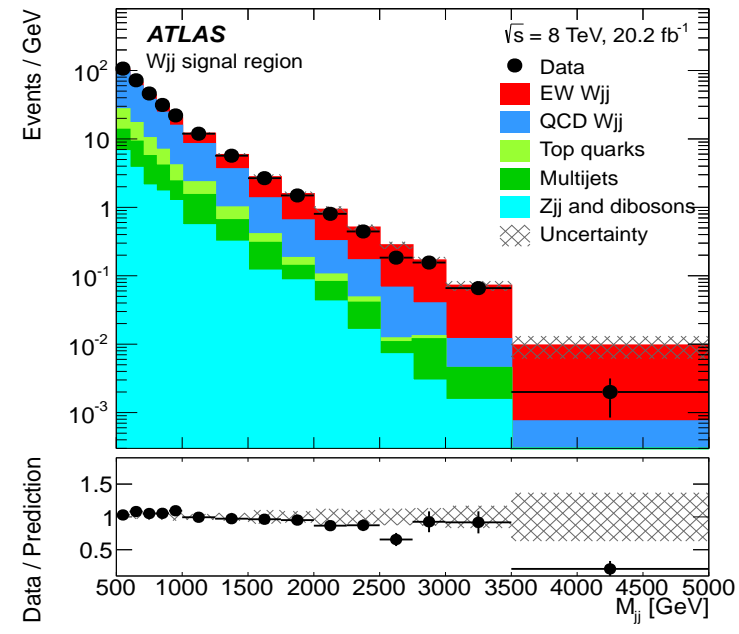

Figure 1: Wjj analysis [2]. Distribution of the dijet invariant mass in the signal region. Data (black dots) is compared to the expectation from the SM (colored histograms).

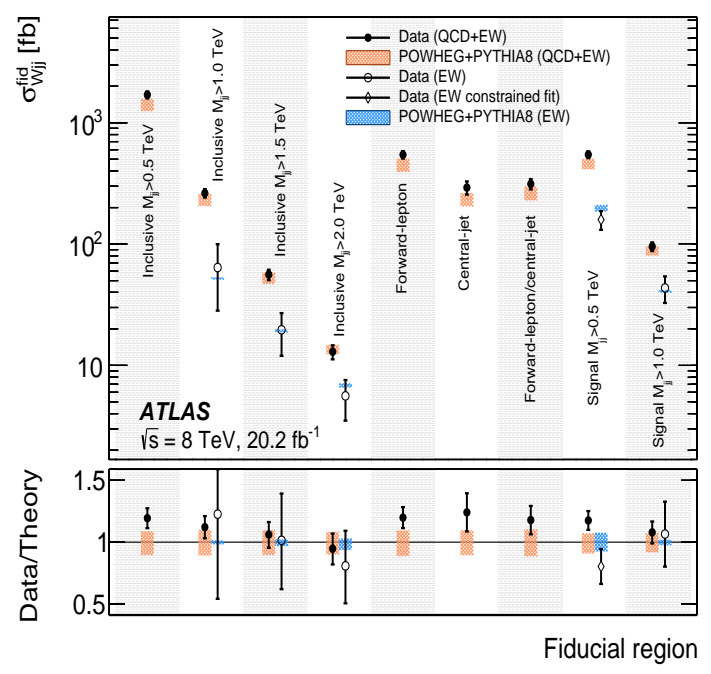

Figure 2: Wjj analysis [2]. Measured fiducial cross sections of the QCD+EW and EW Wjj productions, compared to the expectation from theory. Results in all the regions considered in the analysis are presented. 

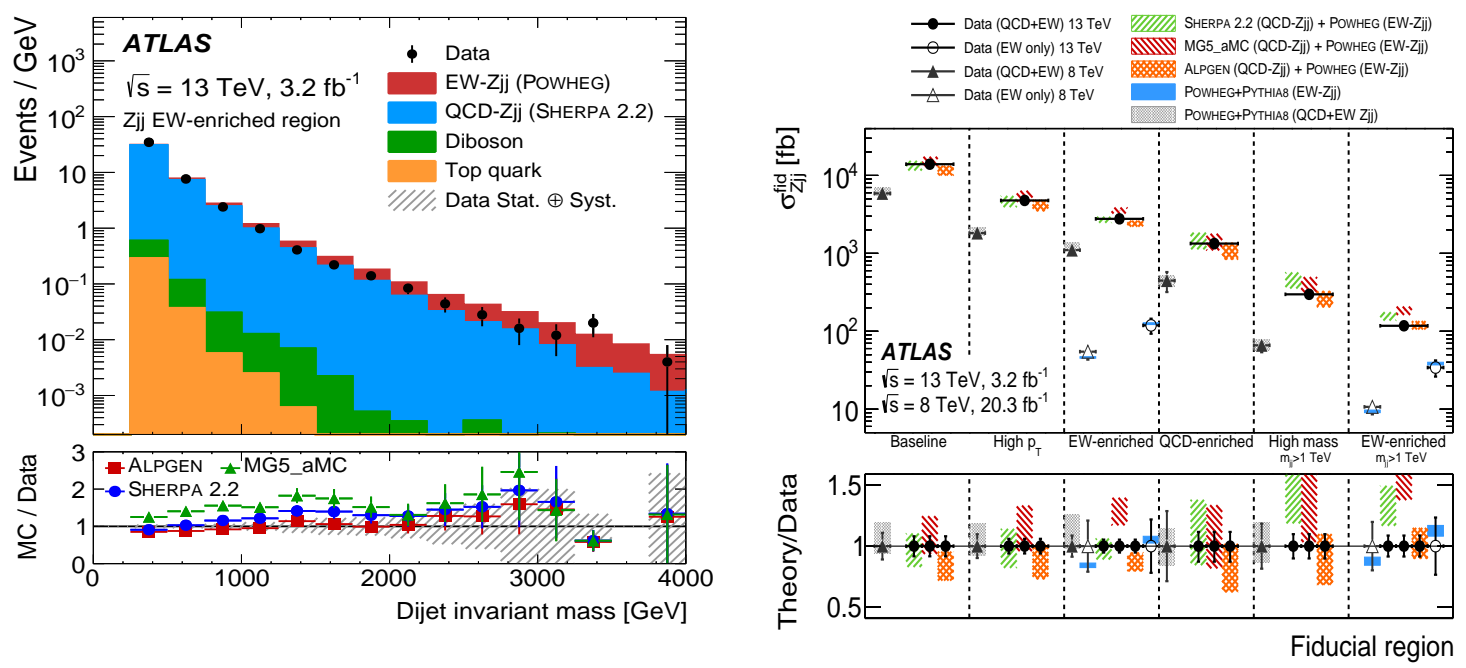

Figure 3: Zjj analysis [3]. Distribution of the dijet invariant mass in the signal region. Data (black dots) is compared to the expectation from the SM (colored histograms).

Figure 4: $\mathrm{Zjj}$ analysis [3]. Measured fiducial cross sections of the QCD+EW and EW Wjj productions, compared to the expectation from theory. Results in all the regions considered in the analysis are presented.

\section{Unfolding of the Wjj and Zjj production cross sections}

Unfolded measurements of the differential $\mathrm{Wjj}$ and $\mathrm{Zjj}$ cross sections have been performed at $8 \mathrm{TeV}$, using a Bayesian iterative technique [5]. Both the EW and QCD plus EW productions were considered, assuming one single lepton flavor.

Unfolded distributions have been determined in all regions of the $\mathrm{Wjj}$ and $\mathrm{Zjj}$ analyses, and for observables distinguishing between the EW and QCD production modes. The $\mathrm{Wjj}$ analysis compares the unfolded measurements to the theoretical predictions from POWHEG+PYHTIA8, SHERPA V1.4.3, and HEJ (for the QCD production), while the $\mathrm{Zjj}$ analysis considers the theoretical predictions from POWHEG+PYHTIA 6 and SHERPA V1.4.3.

As an example, Figure 5 and 6 provide the unfolded distribution of the $\mathrm{Wjj}$ production cross section as function of the leading jet $p_{\mathrm{T}}$, and of the $\mathrm{Zjj}$ production cross section as function of the number of jets in the rapidity gap, respectively.

\section{Constraints on anomalous gauge couplings}

Anomalous gauge couplings have been investigated by the $\mathrm{Wjj}$ and $\mathrm{Zjj}$ analyses at $8 \mathrm{TeV}$. Specific regions derived from the nominal signal regions are considered in order to increase the sensitivity to these couplings.

The $\mathrm{Wjj}$ analysis examines the couplings WWZ and WW $\gamma$ in the context of the aTGC [6] or an EFT [7] model. For this purpose, the SR is modified to enhance events with a large momentum exchange between the incoming partons, which is achieved by rejecting events with a dijet invariant mass and the leading jet $p_{\mathrm{T}}$ below $1 \mathrm{TeV}$ and $600 \mathrm{GeV}$, respectively. The $\mathrm{Zjj}$ analysis probes the 


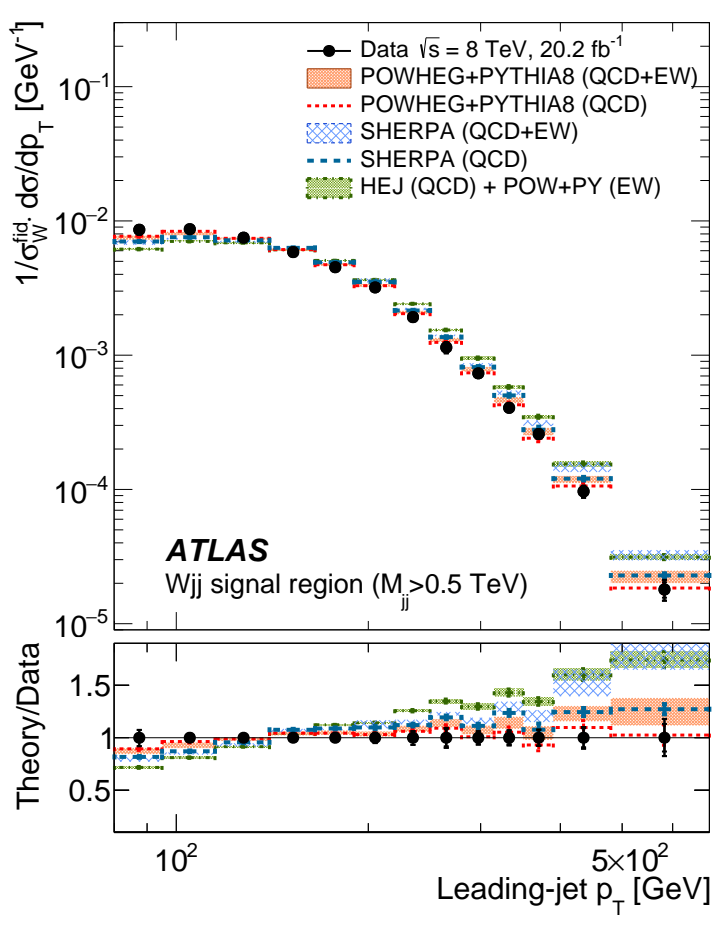

Figure 5: $\mathrm{Wjj}$ analysis [2]. Unfolded QCD and $\mathrm{QCD}+\mathrm{EW}$ cross sections of the $\mathrm{Wjj}$ production as function of the leading jet $p_{\mathrm{T}}$ (in $\mathrm{GeV}$ ). The differential cross section is normalized to the fiducial $\mathrm{Wjj}$ cross section.

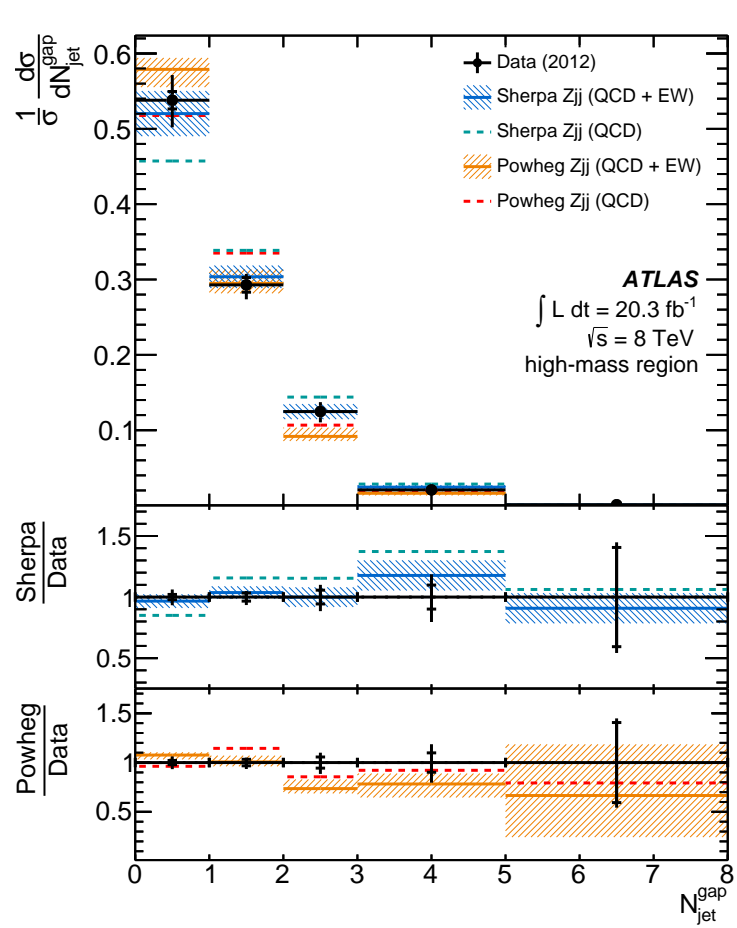

Figure 6: Zjj analysis [3]. Unfolded QCD and QCD+EW cross sections of the $\mathrm{Zjj}$ production as function of the number of jets in the rapidity gap. The differential cross section is normalized to the fiducial $\mathrm{Zjj}$ cross section.

coupling WWZ under the hypothesis of the aTGC model, utilizing a SR with the dijet invariant mass required to exceed $1 \mathrm{TeV}$.

Different suppression scales are tested for the aTGC theory, namely $4 \mathrm{TeV}$ (in the $\mathrm{Wjj}$ analysis only), $6 \mathrm{TeV}$ (in the $\mathrm{Zjj}$ analysis only), and a very large scale (in both analyses, with the $\mathrm{Wjj}$ channel being more sensitive). Among the couplings probed, $\kappa_{Z}, g_{1}^{Z}$, and $\lambda_{V}$ are conserving $C P$ symmetry, while $\tilde{\kappa}_{Z}$ and $\tilde{\lambda}_{V}$ both violate it. Furthermore, in the SM, $\kappa_{Z}$ and $g_{1}^{Z}$ are equal to 1 . Exclusion intervals on $\lambda_{V}$ and the deviations from the SM of $\kappa_{Z}$ and $g_{1}^{Z}$ are provided in Figure 7 and 8 , in the case of a suppression scale of $4 \mathrm{TeV}$.

\section{Conclusion}

The EW and EW plus QCD productions of the $\mathrm{Z}$ boson in association with two jets have been measured by the ATLAS experiment at the LHC using proton-proton collisions at a center of mass energy of 7, 8, and $13 \mathrm{TeV}$, while the EW and EW plus QCD productions of the $\mathrm{W}$ boson in association with two jets have been determined at 7 and $8 \mathrm{TeV}$.

To achieve these results, accurate data-driven techniques have been employed to efficiently suppress systematic uncertainties on the QCD $\mathrm{Wjj}$ and $\mathrm{Zjj}$ productions.

Furthermore, unfolded differential production cross sections at $\sqrt{s}=8 \mathrm{TeV}$ have been estimated for the EW and EW plus QCD processes leading to the Wjj or Zjj final states. 


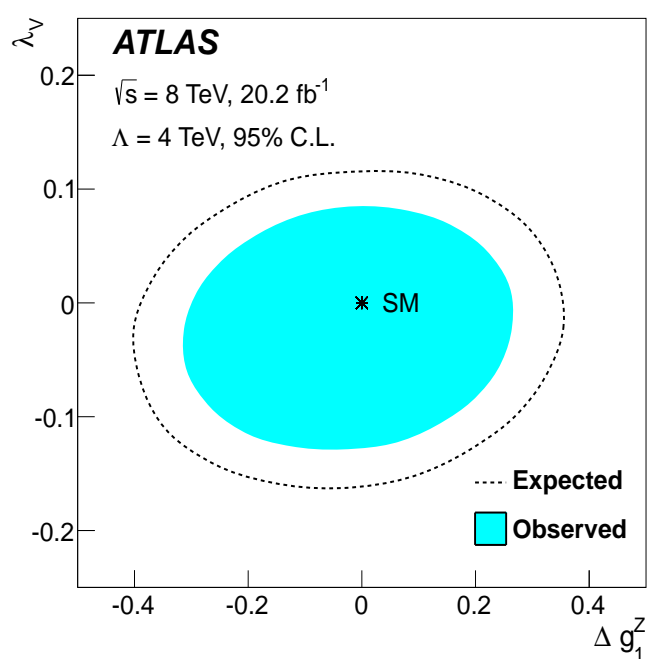

Figure 7: $\mathrm{Wjj}$ analysis [2]. Exclusion limits of the aTGC coupling $\lambda_{V}$ and the deviation from the SM expectation of the aTGC coupling $g_{1}^{Z}$ in a twodimensional plane. The suppression scale is assumed to be $4 \mathrm{TeV}$.

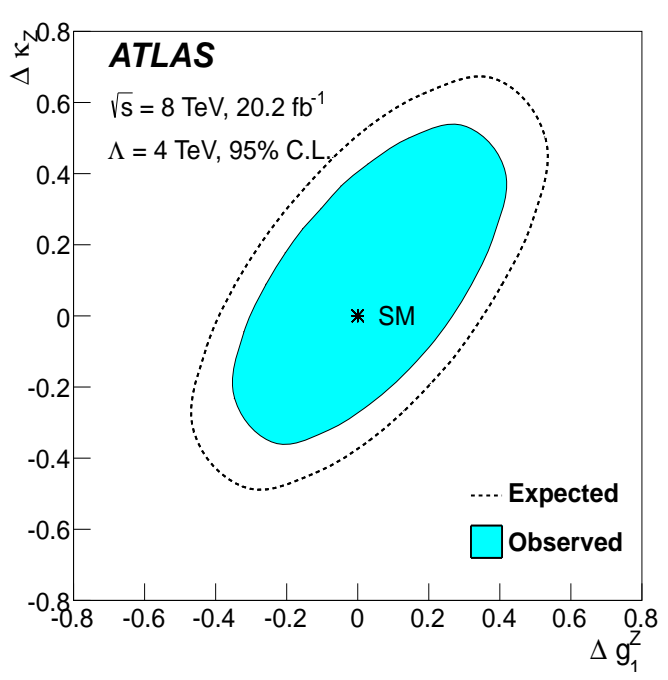

Figure 8: Wij analysis [2]. Exclusion limits of the deviations from the SM expectation of the aTGC couplings $\kappa_{Z}$ and $g_{1}^{Z}$ in a two-dimensional plane. The suppression scale is assumed to be $4 \mathrm{TeV}$.

Finally, stringent constraints have been set on triple-gauge-boson anomalous couplings, which prove to be complementary to the results from the measurement of the WW production [8], in which one off-shell boson is exchanged in the s-channel, instead of two in the t-channel for the VBF Wjj or Zjj productions.

\section{References}

[1] ATLAS Collaboration, The ATLAS experiment at the CERN Large Hadron Collider, J. Instrum. 3, S08003 (2008).

[2] ATLAS Collaboration, Eur. Phys. J. C 77, 474 (2017) [hep-ex/1703.04362].

[3] ATLAS Collaboration, J. High Energy Phys. 04, 031 (2014) [hep-ex/1401.7610].

[4] ATLAS Collaboration, Phys. Lett. B 775, 206 (2017) [hep-ex/1709.10264].

[5] G. D’Agostini, Improved iterative Bayesian unfolding (2010) [physics.data-an/1010.0632].

[6] K. Hagiwara et al, Nucl. Phys. B 282, 253 (1987).

[7] K. Hagiwara et al, Phys. Rev. D 48, 2182 (1993).

[8] ATLAS Collaboration, J. High Energy Phys. 09, 029 (2016) [hep-ex/1603.01702]. 